ANNALS OF “DUNAREA DE JOS” UNIVERSITY OF GALATI

MATHEMATICS, PHYSICS, THEORETICAL MECHANICS

FASCICLE II, YEAR XIII (XLIV) 2021, No. 1

DOI: https://doi.org/10.35219/ann-ugal-math-phys-mec.2021.1.06

\title{
Physico-chemical analysis, systematic benchmarking, and toxicological aspects of the JWH aminoalkylindole class-derived synthetic JWH cannabinoids
}

\author{
Catalina Mercedes Burlacu${ }^{1}$, Adrian Constantin Burlacu, Mirela Praisler ${ }^{1,2}$ \\ 1 "Dunarea de Jos" University of Galati, Faculty of Sciences and Environment, 47 Domneasca \\ Street,800008,Galati,Romania,Catalina.Burlacu@ugal.ro,Mirela.Praisler@ugal.ro \\ ${ }^{2}$ INPOLDE interdisciplinary research network, Dunarea de Jos University of Galati, 111 Domneasca St., \\ 800201 Galati, Romania
}

\begin{abstract}
We are presenting the results obtained by computing different toxicity indices for some of the newest JWH synthetic cannabinoids, by using (Q)SAR models, ADME(T) predictions, simulations of NMR spectral techniques and other different computational dedicated software packages and forensic analytical tools. We have examined the main physical and chemical properties and evaluated the behavioral neurotoxicity and pharmacokinetic profile of 16 aminoalkylindole class-derived synthetic cannabinoids JWH as compared to the Delta-9-tetrahydrocannabinol $(\triangle 9-\mathrm{THC})$, which was chosen as a standard compound. For this purpose, the geometries of the molecules have been optimized by using the AM1 semi-empirical quantum method. The conclusions of a comparative analysis of the toxicities of synthetic and natural cannabinoids are presented.
\end{abstract}

Keywords: JWH synthetic cannabinoids, QSAR descriptors, ADMET prediction.

\section{INTRODUCTION}

Commercial preparations containing synthetic cannabinoids are rapidly emerging as drugs of abuse. Although often assumed to be "safe" and "legal" alternatives to cannabis, reports indicate that synthetic cannabinoids induce toxicity not often associated with the primary psychoactive component of marijuana, $\Delta 9$-tetrahydrocannabinol ( $\Delta 9$-THC).

This paper tried to summarize the evidence that the use of JWH synthetic cannabinoids poses greater health risks relative to marijuana and suggest that distinct pharmacological properties and metabolism of SCBs relative to $\triangle 9$-THC may contribute to this increased toxicity [1].

Physico-chemical analysis of chemicals both in vitro and in vivo is a major research direction, however, it is increasingly considered in recent times that in silico studies in medicine have the potential to accelerate the rate of discovery, while reducing the need for expensive laboratory work and lengthy clinical trials.

In this regard, physico-chemical analysis, biological and toxicological evaluation of synthetic compounds, precursors and derivatives, highlighting patterns of consumption of psychoactive substances, spectral methods of characterization and identification, artificial intelligence, and expert systems are among the most effective methods for the identification, research, and testing of new drugs or emerging chemical compounds, being, at the same time, formidable tools in the fight against trafficking networks of high-risk substance [2].

The present paper provides a review of the main toxic activities of Huffman synthetic cannabinoids probably due to strong CB1 receptor stimulation, as synthetic cannabinoids have a high affinity for the CB1 receptor. 


\section{MATERIALS AND METHODS}

A number of 16 JWH synthetic cannabinoid compounds emerging as drugs of abuse have been characterized and analyzed in comparison with the phytocannabinoid THC ( $\delta$-9tetrahydrocannabinol), most of them part of aminoalkylindole class:

- 7 compounds from naphthylindolines group : JWH-007, JWH-015, JWH-072, JWH-149, JWH-387 JWH-398 and JWH-424;

- 3 compounds from naphthoylpyrroles group: JWH-030, JWH-147 and JWH-370;

- 2 compounds from phenylacetylindoles group: JWH-251 andJWH-302;

- 1 compound from dibenzopyran group: JWH-133;

- 1 hybrid compound from dibenzopyran group: JWH-161;

- 1 compound from naphthylmethylindole group: JWH-184;

- 1 compound from hydrocarbon group: JWH-176.

Relevant literature available online was analyzed and were accessed and queried some of the main online databases, scientific platforms, servers, web benchmarking modules and software packages:

- Online chemical databases: PubChem, Spectrabase, Comptox, The National Institute of Standards and Technology (NIST) and Chemspider.

- Artificial Neural Network Modules and web applications: OCHEM, Comptox, Swiss Institute of Bioinformatics and ADMET Lab scientific platforms.

- QSAR and ADMET standalone Estimation Software Tools and Chemoinformatics Software Suite: HyperChem, Gaussian/GaussView, alvaMolecule, EPA Toxicity Estimation Software Tool (T.E.S.T.), OECD QSAR Toolbox(QSAR) [3].

- Open-Source Programs for Data Visualization and Analysis with Chemical Intelligence: JMol, Osiris Data Warrior.

- Data cleaning and transformation, numerical simulation, statistical modeling and machine learning software package: Python Jupyter Notebook.

The analytical reference methods, the interpretation of the data sets, and the physicochemical and toxicological information available on web databases were in accordance with the regulations and records in the European REACH (Regulation on the Registration, Evaluation, Authorization, and Restriction of Chemicals) database.

The experimental part of the study presented examples and methods applicable in the screening of chemical compounds as an in silico alternative to in vitro and in vivo tests.

For our research, we used the familiar methods of machine learning and QSAR (quantitative structureactivity relationship) activity, Artificial Neural Network (ANN), and two simulated methods of spectral identification 1H-NMR and 13C-NMR [4].

The geometries of the molecules of the 16 target synthetic cannabinoids and the THC compound downloaded in the MOL file format have been optimized with HyperChem, and Gaussian/ GaussView software packages by using the AM1 semi-empirical quantum method (e.g. Figure 1), starting from the canonical SMILES molecular identifiers from the PubChem scientific platform and the molecules' structures downloaded in the MOL file format(.mol).

The small molecules obtained after optimization were saved again in MOL format (.mol) and then were compiled into output files MDL (.sdf) and in a tabular format CSV (.csv) with alvaMolecule for extended portability to other software packages.

Most of the 75 QSAR and the 40 ADMET molecular descriptors calculated and tested in our study have been selected from three blocks: constitutional descriptors, molecular properties, and indices drug likeness (the complete list of calculated and tested molecular descriptors can be downloaded on request). We prepared a synopsis, tables, and comparative diagrams for each of the 16 synthetic cannabinoids and THC containing physico-chemical and toxicological data and information obtained from online databases, validated and scientifically accepted $[5,6]$. 


\section{RESULTS AND DISCUSSION}

The ADMET predictions were performed for all the studied compounds.

Table 1. AQUEOUS SOLUBILITY(logS Descriptor) EVALUATION REPORT

\begin{tabular}{|c|c|c|}
\hline \multicolumn{3}{|c|}{$\begin{array}{c}\text { AQUEOUS SOLUBILITY (LOGS DESCRIPTOR) } \\
\text { EVALUATION REPORT }\end{array}$} \\
\hline COMPOUND & IDENTIFIER & $\begin{array}{c}\text { Log S(SILICOS-IT) } \\
\log \text { mol/ L }\end{array}$ \\
\hline 01 & THC & -3.500 \\
\hline 02 & JWH 007 & -6.346 \\
\hline 03 & JWH 015 & -6.264 \\
\hline 04 & JWH 030 & -6.177 \\
\hline 05 & JWH 072 & -6.414 \\
\hline 06 & JWH 133 & -6.086 \\
\hline 07 & JWH 147 & -6.446 \\
\hline 08 & JWH 149 & -6.410 \\
\hline 09 & JWH 161 & -6.191 \\
\hline 10 & JWH 176 184 & -7.319 \\
\hline 11 & JWH 184 & -6.941 \\
\hline 12 & JWH 251 & -6.444 \\
\hline 13 & JWH 302 & -6.357 \\
\hline 14 & JWH 370 & -6.427 \\
\hline 15 & JWH 387 & -6.495 \\
\hline 16 & JWH 398 & -6.490 \\
\hline 17 & JWH 424 & -6.458 \\
\hline & &
\end{tabular}

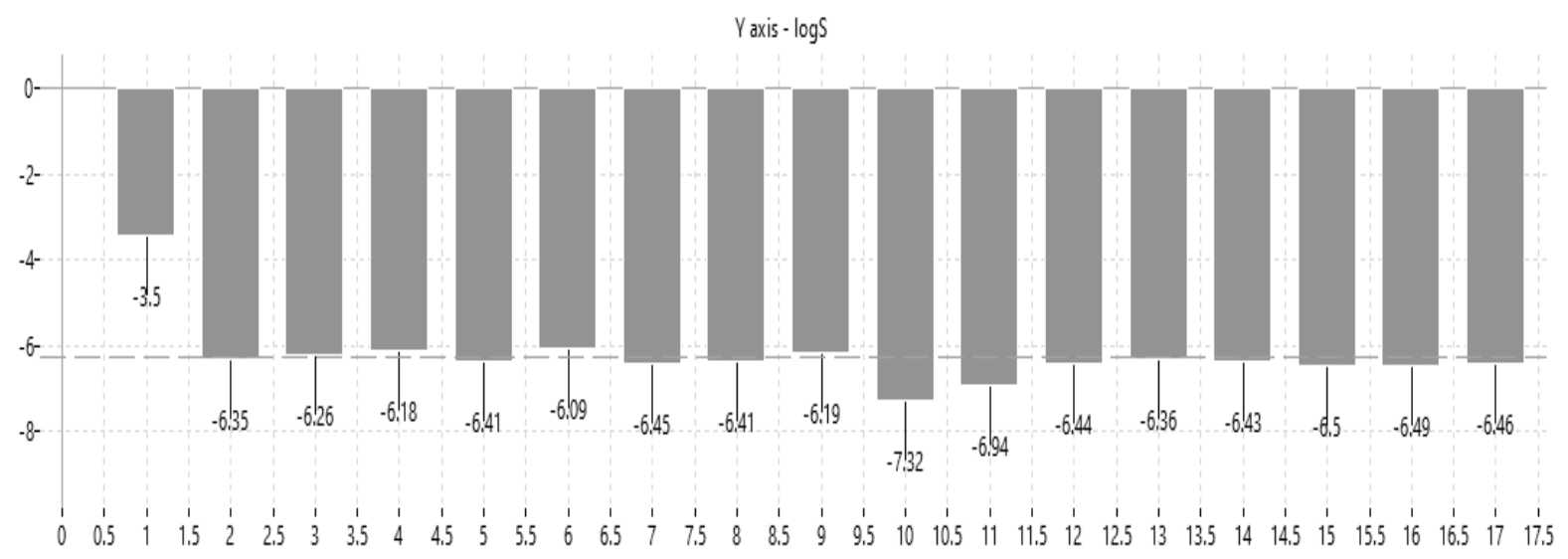

Figure 1. AQUEOUS SOLUBILITY (logS) descriptor (chart drafted with alvaMolecule software package)

Table 1 presents the AQUEOUS SOLUBILITY determined for the targeted compounds, a property that significantly affects the absorption and distribution characteristics of a drug. Most of the drugs on the market have an estimated $\operatorname{logS}$ value of more than -4 . Typically, a low solubility is associated with bad absorption. Therefore, poorly soluble compounds are usually avoided [7].

From the examination of the charts and the ADMET evaluation report regarding the approximate $\operatorname{logS}$ parameter for the $16 \mathrm{JWH}$ synthetic cannabinoids presented and for the standard reference phytocannabinoid THC, we draw the following conclusions:

- The approximate value, in silico, of the $\operatorname{logS}$ parameter for the THC phytocannabinoid, is 3,500 and is, therefore, higher than the general average of the experimentally calculated values, of -4 , the phytocannabinoid having a high solubility in water, being, therefore, optimal. 
- None of the approximate values for the 16 synthetic cannabinoids analyzed was in the optimal range of water solubility, which was much lower compared to both the approximate value, in silico, for THC and the general average calculated experimentally in around -4 .

- The highest value of the 16 synthetic cannabinoids JWH had the compound JWH 133 ($6,086)$, thus having a higher water solubility than the other chemical compounds analyzed, and the lowest value was approximated for JWH $176(-7,319)$.

\begin{tabular}{|c|c|c|}
\hline \multicolumn{3}{|c|}{$\begin{array}{c}\text { LOGD (DISTRIBUTION COEFFICIENT D AT PH=7.4) } \\
\text { (LOGD DESCRIPTOR) EVALUATION REPORT }\end{array}$} \\
\hline COMPOUND & IDENTIFIER & $\begin{array}{c}\text { logD } \\
\text { (OCTANOL/WATER) }\end{array}$ \\
\hline 01 & THC & 2.007 \\
\hline 02 & JWH 007 & 3.470 \\
\hline 03 & JWH 015 5.245 \\
\hline 04 & JWH 030 & 3.052 \\
\hline 05 & JWH 072 & 3.061 \\
\hline 06 & JWH 133 3.448 \\
\hline 07 & JWH 147 & 3.563 \\
\hline 08 & JWH 149 & 3.533 \\
\hline 09 & JWH 161 & 2.190 \\
\hline 10 & JWH 176 & 3.553 \\
\hline 11 & JWH 184 & 3.443 \\
\hline 12 & JWH 251 & 3.433 \\
\hline 13 & JWH 302 & 2.033 \\
\hline 14 & JWH 370 & 3.538 \\
\hline 15 & JWH 387 & 3.364 \\
\hline 16 & JWH 398 & 3.188 \\
\hline 17 & JWH 424 & 3.282 \\
\hline
\end{tabular}

Table 2. $\log$ D (DISTRIBUTION COEFFICIENT D AT PH=7.4) (LogD Descriptor)

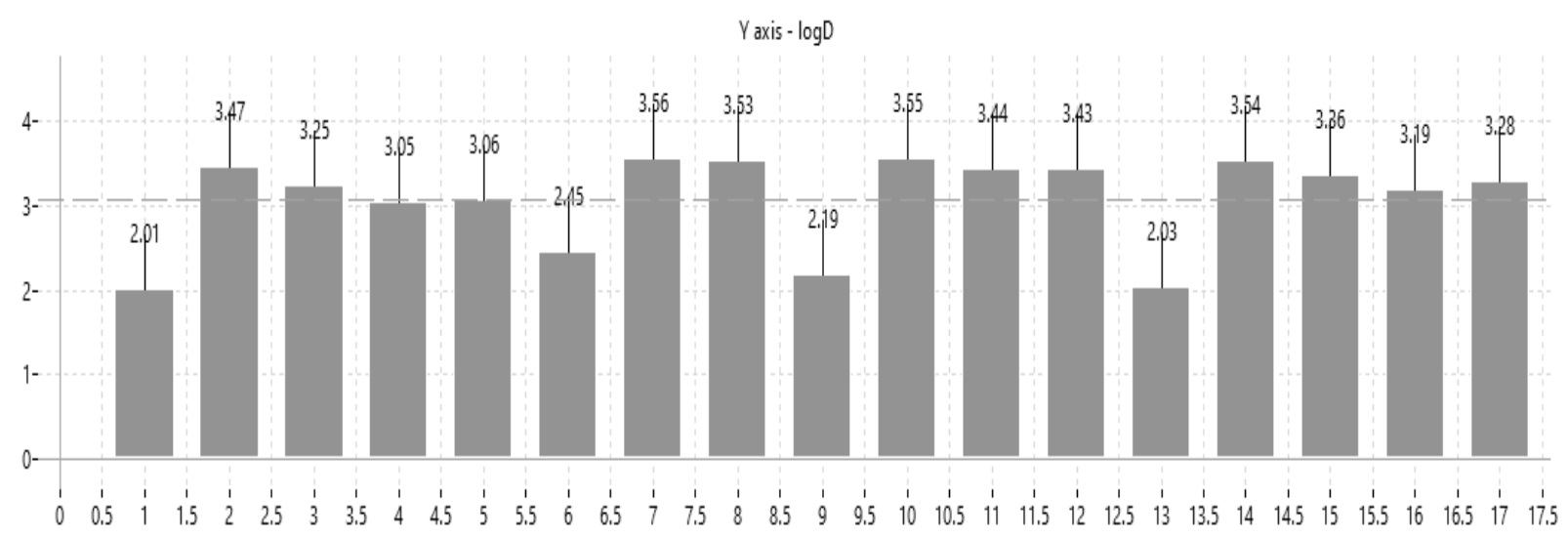

Figure 2. $\log D$ (DISTRIBUTION COEFFICIENT D AT PH=7.4) (LogD) descriptor (chart drafted with alvaMolecule software package) EVALUATION REPORT

$\log \mathrm{D}$, the distribution constant is a better descriptor of the lipophilicity of a molecule. This can be determined in a similar way to $\log$, but instead of using water, the aqueous phase is adjusted to a specific $\mathrm{pH}$ using a buffer [8]. 
Therefore, $\log \mathrm{D}$ is $\mathrm{pH}$ dependent, so the $\mathrm{pH}$ at which $\log \mathrm{D}$ was measured must be specified. Of particular interest is $\log \mathrm{D}$ at $\mathrm{pH}=7.4$ (physiological $\mathrm{pH}$ of blood serum).

According to Table 2, the approximate values can be interpreted as follows:

- 1 to 3: Moderate solubility; Moderate permeability; Low metabolism.

- 3 to 5: Low solubility; High permeability; Moderate to high metabolism.

From the examination of the graphs and the $\operatorname{ADME(T)}$ evaluation report regarding the approximate $\log \mathrm{D}$ parameter, we draw the following conclusions:

- The approximate value, in silico, of the $\log \mathrm{D}$ parameter for the THC phytocannabinoid is 2.007 and is therefore between 1 and 3, this aspect can be interpreted as having moderate solubility, moderate permeability and low metabolism, the phytocannabinoid being within the values of other abusers / drugs on the market and having the lowest value of all JWH synthetic cannabinoids analyzed in this paper.

- Only 3 of the 16 synthetic cannabinoids JWH analyzed comparatively still have a value between 1-3, so moderate solubility, moderate permeability, and low metabolism, JWH $133(2,448)$, JWH 161 (2,190), and JWH 302 (2,033).

- The remaining 13 JWH synthetic cannabinoids analyzed having values between $3-5$, so low solubility, high permeability, moderate to high metabolism, the highest value having JWH 147 $(3,563)$.

The $\log \mathrm{P}$ value of a compound, which is the logarithm of its partition coefficient between $\mathrm{n}$ octanol and log water (c octanol / c water), is a well-established measure of the hydrophilicity of the compound.

High $\log \mathrm{P}$ values result in low absorption or permeability. It has been shown that the compounds have a reasonable property of being well absorbed, their $\log \mathrm{P}$ value should not exceed 5.0.

Table 3. $\log$ P (DISTRIBUTION COEFFICIENT P)(logP Descriptor) EVALUATION REPORT

\begin{tabular}{|c|c|c|c|}
\hline \multicolumn{4}{|c|}{$\begin{array}{l}\text { OGP (DISTRIBUTION COEFFICIENT P) } \\
\text { (LOGP DESCRIPTOR) EVALUATION REPORT }\end{array}$} \\
\hline COMPOUND & IDENTIFIER & LOGP & $\begin{array}{c}\text { TOTAL } \\
\text { MOL WEIGHT }\end{array}$ \\
\hline 01 & THC & 5.736 & 314.467 \\
\hline 02 & JWH 007 & 6.524 & 355.480 \\
\hline 03 & JWH 015 & 5.744 & 327.426 \\
\hline 04 & JWH 030 & 5.063 & 291.393 \\
\hline 05 & JWH 072 & 5.436 & 313.399 \\
\hline 06 & JWH 133 & 6.375 & 312.495 \\
\hline 07 & JWH 147 & 7.120 & 381.517 \\
\hline 08 & JWH 149 & 6.833 & 369.506 \\
\hline 09 & JWH 161 & 7.301 & 403.564 \\
\hline 10 & JWH 176 & 7.358 & 324.466 \\
\hline 11 & JWH 184 & 6.884 & 341.496 \\
\hline 12 & JWH 251 & 5.565 & 319.447 \\
\hline 13 & JWH 302 & 5.266 & 335.446 \\
\hline 14 & JWH 370 & 7.038 & 381.517 \\
\hline 15 & JWH 387 & 6.978 & 420.349 \\
\hline 16 & JWH 398 & 6.869 & 375.898 \\
\hline 17 & JWH 424 & 6.978 & 420.349 \\
\hline
\end{tabular}




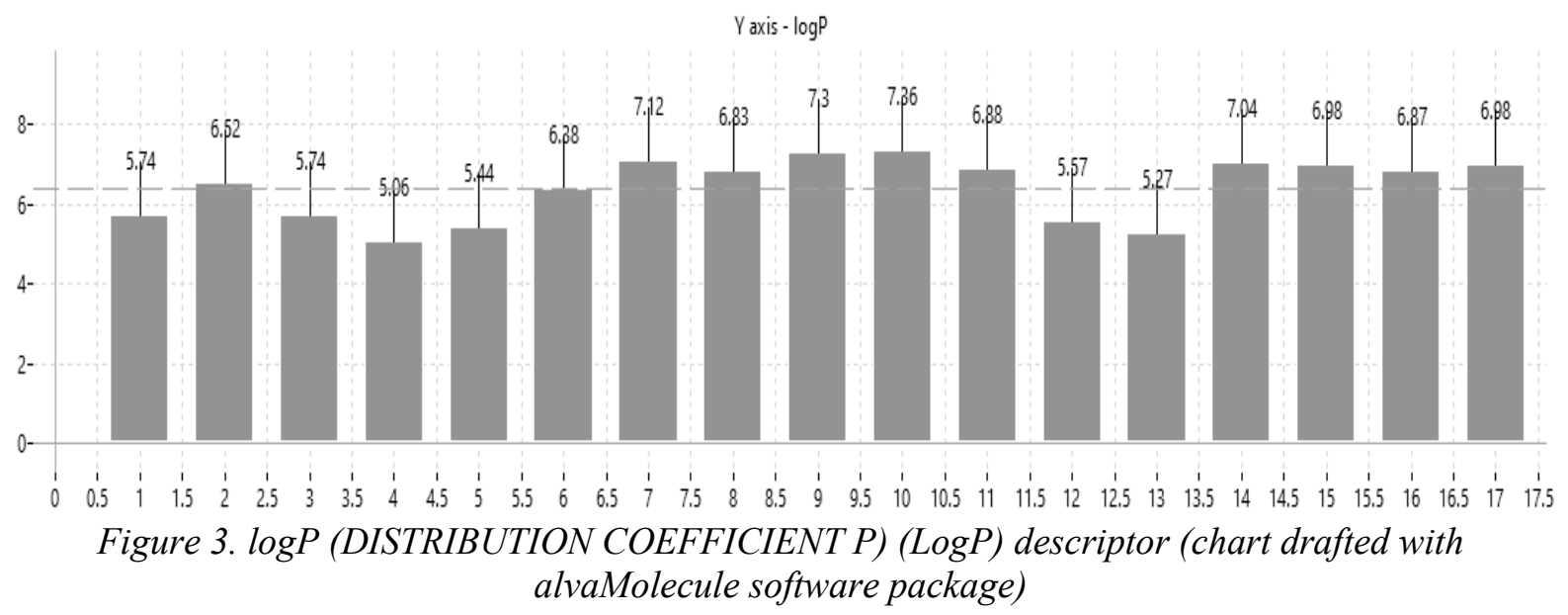

Lipophilicity is probably the most important physicochemical property of a potential drug, it plays a role in solubility, absorption, membrane penetration, plasma protein binding, distribution, CNS penetration, and partitioning into other tissues or organs such as the liver and has an impact on escape routes [8].

From the examination of the charts and the ADMET evaluation report regarding the approximate $\log \mathrm{P}$ parameter, we draw the following conclusions:

- The approximate value, in silico, of the $\log \mathrm{P}$ parameter for the THC phytocannabinoid is 5,736 and therefore, is higher than the overall average of the experimentally calculated values of 5,0, the phytocannabinoid having an absorption or a low permeability to the average of the other abuse / medicines on the market.

- Of the 16 synthetic cannabinoids JWH analyzed in comparison, 4 compounds have a lower $\log$ P value than THC and therefore have a better absorption or permeability than THC, namely, in order, JWH $030(5,063)$, JWH $302(5,266)$, JWH $072(5,436)$ and JWH $251(5,565)$.

- None of the approximate values for the 16 synthetic cannabinoids analyzed were in the optimal range of absorption, or of reduced permeability having higher values, compared to the general average calculated experimentally around 5.0.

- The lowest value of the 16 JWH synthetic cannabinoids was compound JWH $030(5,063)$, thus having a good absorption or permeability than the other chemical compounds analyzed, and the highest value was approximated for JWH 176.

Caco-2 promiscuity parameter is used for toxicity assessments and refers to a human colon epithelial cancer cell line that is used to model human intestinal absorption of drugs. Enzyme promiscuity refers to the ability of an enzyme to catalyze a fortuitous side reaction besides its main reaction [9].

This parameter is evaluated based on the logPAPP parameter, i.e. the in vitro passive membrane permeability across the Caco-2 cell. 
Table 4. $\log$ PAPP (CACO-2 PERMEABILITY)(Caco-2 Descriptor) EVALUATION REPORT

\begin{tabular}{|c|c|c|}
\hline \multicolumn{3}{|c|}{ LOGPAPP (CACO-2 PERMEABILITY) } \\
\hline COMPO-2 DESCRIPTOR) EVALUATION REPORT \\
\hline 01 & IDENTIFIER & CACO2( CM/S) \\
\hline 02 & THC & -4.746 \\
\hline 03 & JWH 007 & -4.628 \\
\hline 04 & JWH 015 & -4.547 \\
\hline 05 & JWH 030 & -4.452 \\
\hline 06 & JWH 072 & -4.539 \\
\hline 07 & JWH 133 & -4.548 \\
\hline 08 & JWH 147 & -4.718 \\
\hline 09 & JWH 149 & -4.616 \\
\hline 10 & JWH 161 & -4.966 \\
\hline 11 & JWH 176 & -4.500 \\
\hline 12 & JWH 184 & -4.598 \\
\hline 13 & JWH 251 & -4.572 \\
\hline 14 & JWH 302 & -4.641 \\
\hline 15 & JWH 370 & -4.727 \\
\hline 16 & JWH 387 & -4.635 \\
\hline 17 & JWH 398 & -4.622 \\
\hline & JWH 424 & -4.635 \\
\hline
\end{tabular}

The results obtained regarding the Caco-2 promiscuity of the studied JWH cannabinoids are presented in Table 4. The largest logPapp value, second only to that determined for the natural THC compound, was obtained for JWH-161.

Yaxis - caco2

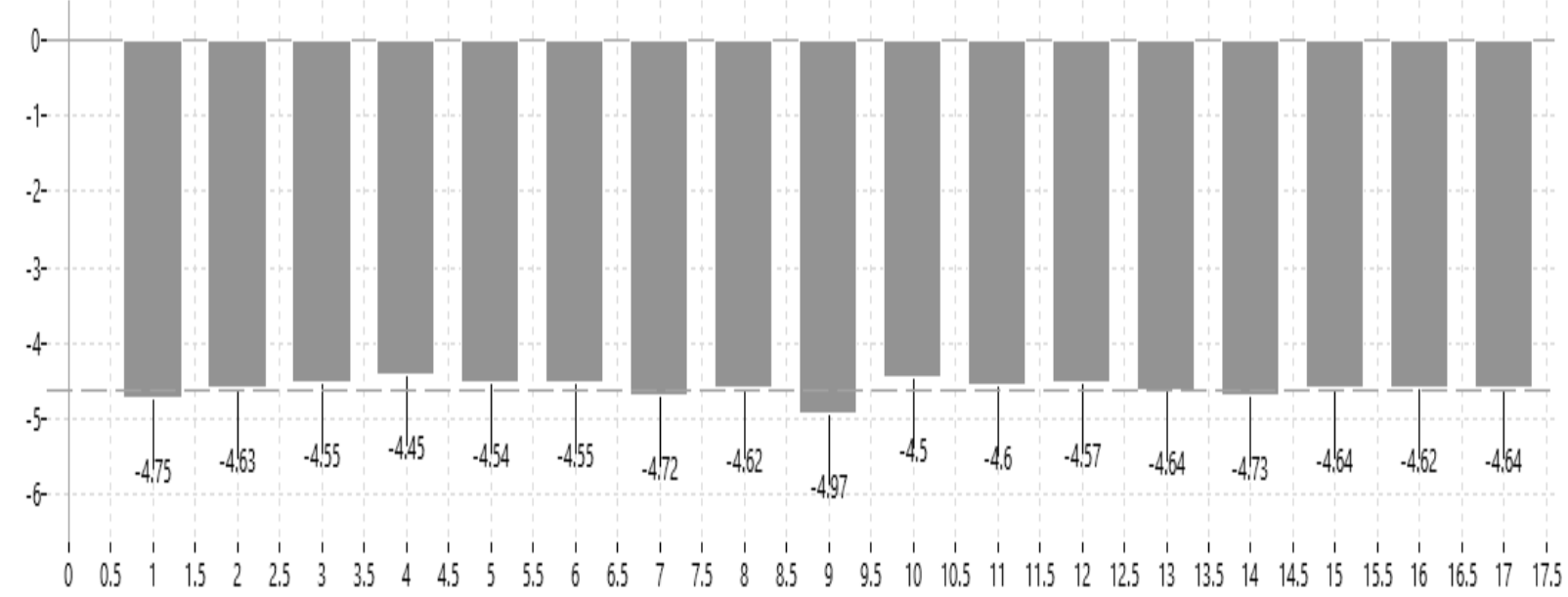

Figure 4. $\log P A P P$ (CACO-2 PERMEABILITY) (Caco-2) descriptor (chart drafted with alvaMolecule software package) 
Table 5. BBB (Blood-Brain Barrier) (BBB+ Descriptor) EVALUATION REPORT

\begin{tabular}{|c|c|c|c|}
\hline \multicolumn{4}{|c|}{$\begin{array}{c}\text { BBB (BLOOD-BRAIN BARRIER) (BBB+ DESCRIPTOR) } \\
\text { EVALUATION REPORT }\end{array}$} \\
\hline COMPOUND & IDENTIFIER & $\begin{array}{c}\text { CATEGORY } \\
\text { 1-PERMEABLE/0- NONPERMEABLE }\end{array}$ & PROBABILITY \\
\hline 01 & THC & 1 & 0.878 \\
\hline 02 & JWH 007 & 1 & 0.969 \\
\hline 03 & JWH 015 & 1 & 0.978 \\
\hline 04 & JWH 030 & 1 & 0.882 \\
\hline 05 & JWH 072 & 1 & 0.950 \\
\hline 06 & JWH 133 & 1 & 0.974 \\
\hline 07 & JWH 147 & 1 & 0.882 \\
\hline 08 & JWH 149 & 1 & 0.980 \\
\hline 09 & JWH 161 & 1 & 0.950 \\
\hline 10 & JWH 176 & 1 & 0.973 \\
\hline 11 & JWH 184 & 1 & 0.990 \\
\hline 12 & JWH 251 & 1 & 0.904 \\
\hline 13 & JWH 302 & 1 & 0.909 \\
\hline 14 & JWH 370 & 1 & 0.857 \\
\hline 15 & JWH 387 & 1 & 0.918 \\
\hline 16 & JWH 398 & 1 & 0.959 \\
\hline 17 & JWH 424 & 1 & 0.854 \\
\hline
\end{tabular}

Table 5 presents the results obtained regarding the blood-brain barrier $\mathrm{BBB}+$. This semipermeable membrane barrier is highly selective and separates the circulating blood from the brain and extracellular fluid in the central nervous system (CNS).

The blood-brain barrier works effectively to protect the brain from the circulation of pathogens. Consequently, infections caused by the blood of the brain are rare, and when they occur, they are very difficult to treat.

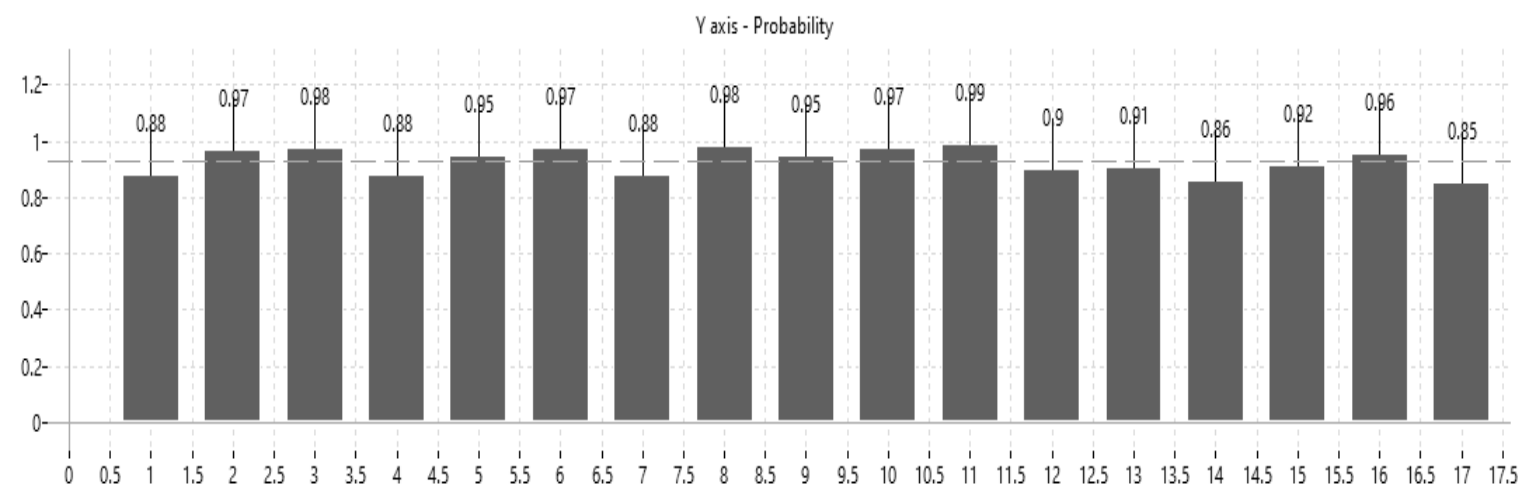

Figure 5. BBB (Blood-Brain Barrier) (BBB+) descriptor (chart drafted with alvaMolecule software package)

The blood-brain barrier becomes more permeable during inflammation. In some cases, a drug should be given directly into the cerebrospinal fluid (CSF), where it can enter the brain by crossing the blood-fluid barrier cerebrum. 
From this point of view, Table 5 indicates that all the studied JWH cannabinoids have a significantly higher probability than THC, their natural counterpart. Hence, we may conclude that all these compounds are indeed more toxic for the brain than the latter compound. This effect may arise from a single or from multiple exposures in less than 24 hours. The toxicity is considered acute if the adverse effects occur in less than 14 days after the administration of the compound.

Table 6 indicates that all the studied JWH cannabinoids have a higher LD50 than THC with the exception of JWH-161 and JWH-424. A lower LD50 is indicative of increased toxicity.

Table 6. LD50 (LD50 of acute toxicity) (LD50 Descriptor) EVALUATION REPORT

\begin{tabular}{|c|c|c|}
\hline \multicolumn{3}{|c|}{$\begin{array}{c}\text { LD50 (LD50 OF ACUTE TOXICITY) } \\
\text { (LD50 DESCRIPTOR) EVALUATION REPORT }\end{array}$} \\
\hline COMPOUND & IDENTIFIER & $\begin{array}{c}\text { LD50 } \\
\text { (-LOG MOL / KG) }\end{array}$ \\
\hline 01 & THC & 2.666 \\
\hline 02 & JWH 007 & 2.547 \\
\hline 03 & JWH 015 & 2.604 \\
\hline 04 & JWH 030 & 2.441 \\
\hline 05 & JWH 072 & 2.519 \\
\hline 06 & JWH 133 & 2.306 \\
\hline 07 & JWH 147 & 2.592 \\
\hline 08 & JWH 149 & 2.560 \\
\hline 09 & JWH 161 & 2.861 \\
\hline 10 & JWH 176 & 2.652 \\
\hline 11 & JWH 184 & 2.525 \\
\hline 12 & JWH 251 & 2.549 \\
\hline 13 & JWH 302 & 2.478 \\
\hline 14 & JWH 370 & 2.574 \\
\hline 15 & JWH 387 & 2.629 \\
\hline 16 & JWH 398 & 2.672 \\
\hline 17 & JWH 424 & 2.706 \\
\hline
\end{tabular}

Tetrahymena pyriformis toxicity (TPT) is the most commonly ciliated model used as a toxicology endpoint. The results obtained for the targeted JWH cannabinoids are presented in Table 7.

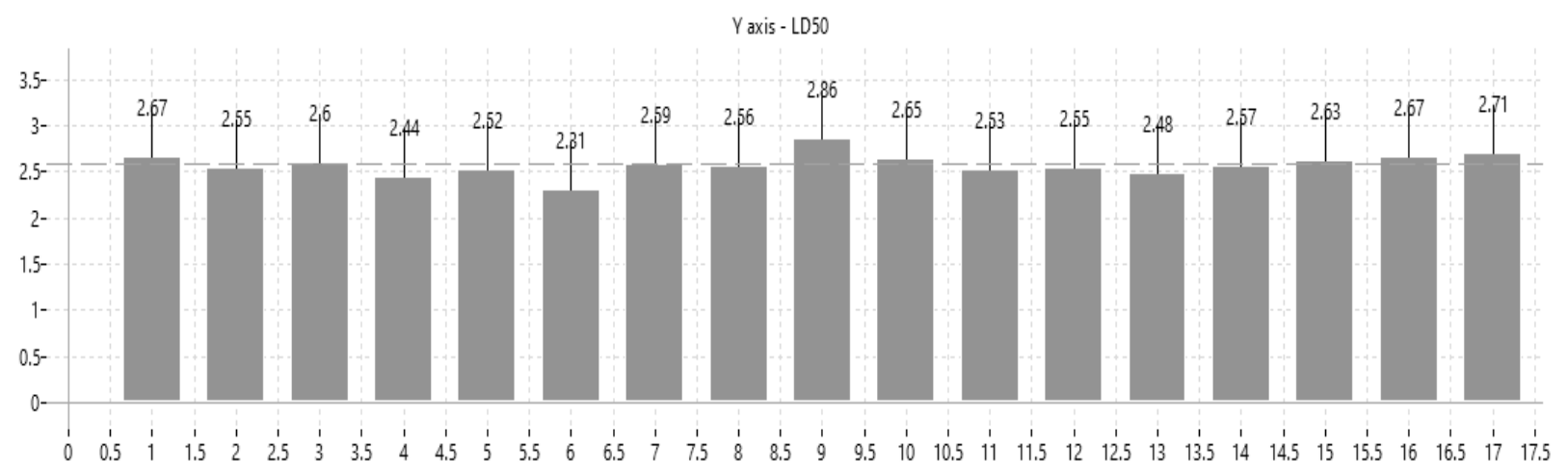

Figure 6. LD50 (LD50 of acute toxicity) (LD50) descriptor (chart drafted with alvaMolecule software package) 
Table 7. TETRAHYMENA PYRIFORMIS TOXICITY (logIGC50-1 Descriptor) EVALUATION REPORT

\begin{tabular}{|c|c|c|}
\hline \multicolumn{3}{|c|}{$\begin{array}{l}\text { LOGIGC50-1 TETRAHYMENA PYRIFORMIS TOXICITY } \\
\text { (LOGIGC50-1 DESCRIPTOR) } \\
\text { EVALUATION REPORT }\end{array}$} \\
\hline COMPOUND & IDENTIFIER & LOG(IGC50-1) (MODEL 3) \\
\hline 01 & THC & 2.3 \\
\hline 02 & JWH 007 & 2.8 \\
\hline 03 & JWH 015 & 2.3 \\
\hline 04 & JWH 030 & 2.5 \\
\hline 05 & JWH 072 & 2.1 \\
\hline 06 & JWH 133 & 1.9 \\
\hline 07 & JWH 147 & 2.9 \\
\hline 08 & JWH 149 & 2.8 \\
\hline 09 & JWH 161 & 2.8 \\
\hline 10 & JWH 176 & 2.7 \\
\hline 11 & JWH 184 & 3.1 \\
\hline 12 & JWH 251 & 2.6 \\
\hline 13 & JWH 302 & 2.6 \\
\hline 14 & JWH 370 & 2.9 \\
\hline 15 & JWH 387 & 2.5 \\
\hline 16 & JWH 398 & 2.8 \\
\hline 17 & JWH 424 & 2.4 \\
\hline & & \\
\hline
\end{tabular}

The toxicity data is expressed as the negative logarithm of $50 \%$ growth inhibitory concentration (pIGC50) values, the threshold value being pIGC50 $=-0.5$. In other words, the compounds having

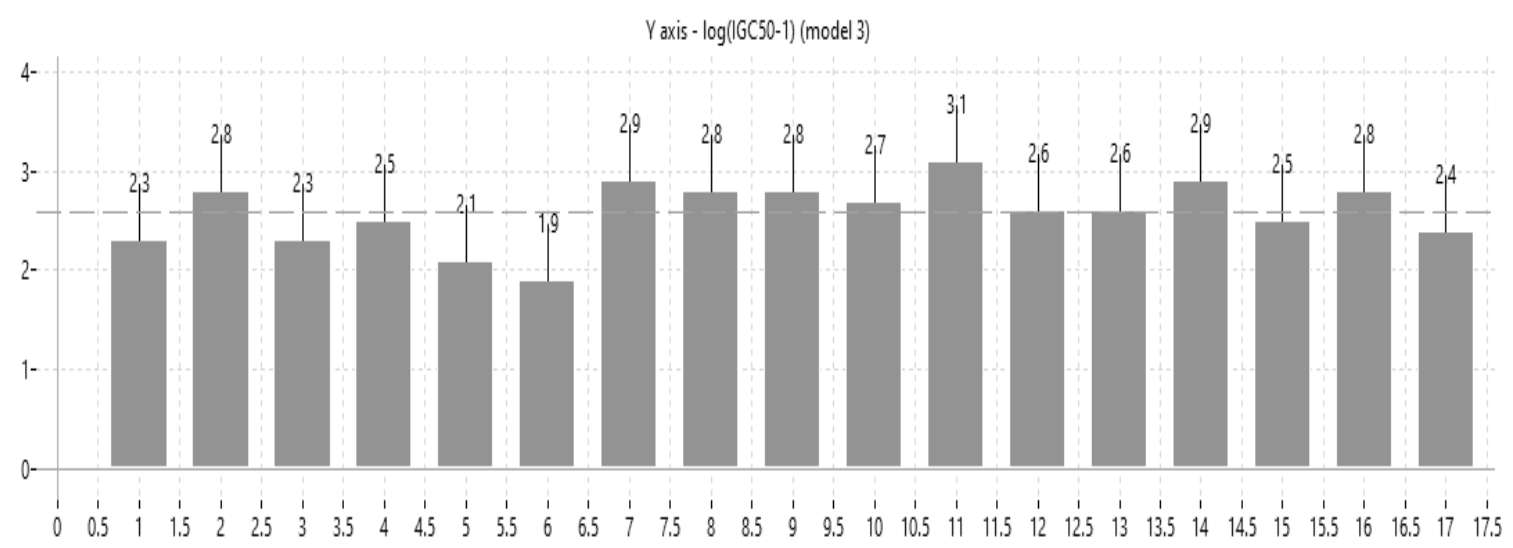

Figure 7. logIGC50-1 TETRAHYMENA PYRIFORMIS TOXICITY descriptor (chart drafted with alvaMolecule software package)

pIGC50 $>-0.5$ are assigned as TPT and the others as non-TPT. The results obtained for the AMES mutagenicity are presented in Table 8. 
Table 8. AMES MUTAGENICITY(Ames Mutagenicity Descriptor) EVALUATION REPORT

\begin{tabular}{|c|c|c|c|}
\hline \multicolumn{5}{|c|}{$\begin{array}{c}\text { AMES MUTAGENICITY } \\
\text { (AMES MUTAGENICITY DESCRIPTOR) } \\
\text { EVALUTION REPORT }\end{array}$} \\
\hline \multirow{2}{*}{ COMPOUND } & IDENTIFIER & $\begin{array}{c}\text { CATEGORY } \\
\text { 1-ACTIVE/ 0-INACTIVE }\end{array}$ & PROBABILITY \\
\hline 01 & THC & 0 & 0.090 \\
\hline 02 & JWH 007 & 1 & 0.554 \\
\hline 03 & JWH 015 & 1 & 0.560 \\
\hline 04 & JWH 030 & 1 & 0.504 \\
\hline 05 & JWH 072 & 0 & 0.628 \\
\hline 06 & JWH 133 & 0 & 0.084 \\
\hline 07 & JWH 147 & 0 & 0.498 \\
\hline 08 & JWH 149 & 0 & 0.530 \\
\hline 09 & JWH 161 & 1 & 0.316 \\
\hline 10 & JWH 176 & 0 & 0.484 \\
\hline 11 & JWH 184 & 1 & 0.700 \\
\hline 12 & JWH 251 & 1 & 0.480 \\
\hline 13 & JWH 302 & 0 & 0.508 \\
\hline 14 & JWH 370 & 0 & 0.506 \\
\hline 15 & JWH 387 & 0 & 0.488 \\
\hline 16 & JWH 398 & 0.494 \\
\hline 17 & JWH 424 & 0.488 \\
\hline
\end{tabular}

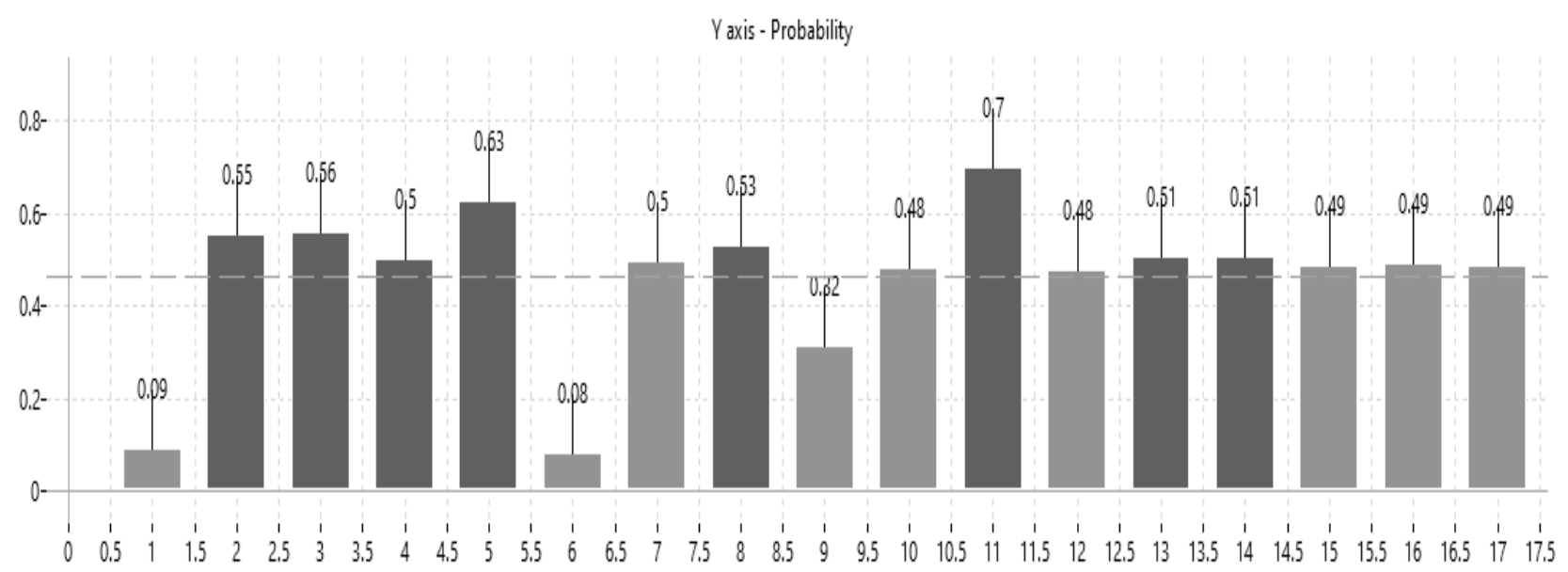

Figure 8. AMES MUTAGENICITY(Ames Mutagenicity) descriptor descriptor (chart drafted with alvaMolecule software package)

They clearly indicate that THC does not exhibit any important mutagenic activity, while some of the JWH cannabinoids exhibit immunotoxicity activity as a priority action. Many other parameters were studied in this research with important results [9]. 


\section{CONCLUSIONS}

The comparative results of the in silico tests confirm that some JWH synthetic cannabinoids are even more toxic than their natural counterpart. All JWH synthetic cannabinoids analyzed have much more toxic potential, having a high affinity for the CB1 receptor. Some have a particularly low value of the Ki constant binding to more than 100 times more closely to the CB1 receptor than THC. JWH synthetic cannabinoids can be confirmed and analyzed using artificial intelligence, expert systems, and spectral techniques such as GC-MS, GC-IR, GC-FTIR, FT-IR, ATR-FTIR, and RAMAN which are among the most efficient and formidable tools in the fight against high-risk trafficking networks [10].

\section{References}

1. Praisler M., Coman M.M., Gosav S., Study regarding the toxicological activity of some JWH synthetic cannabinoids, Annals of the Dunarea de Jos University of Galati, Fasc. II, 1 (2018) 25-32.

2. Praisler M., Ciochină Ș., Pattern recognition analysis of the class identity recognition efficiency of a portable laser infrared sensor detecting amphetamines and their main precursors; chemometric application operating a portable laser infrared sensor detecting illicit phenethylamines, Annals of the Dunarea de Jos University of Galati,, Fasc. II, 1 (2019) 55-62.

3. Todeschini R., Consonni V., Molecular Descriptors for Chemoinformatics, (Mannhold R., Kubinyi H., Folkers G., Weinheim, Wiley-VCH editors), 2009.

4. Praisler M., Ion A., Gosav S., Choosing relevant functional groups for optimizing Artificial Neural Networks detecting NBOMe hallucinogens, Annals of the Dunarea de Jos University of Galati, Fasc. II, 1 (2019) 160-166.

5. Praisler M., Gosav S., Birsa M.L., Principal Component Analysis Coupled with Artificial Neural Networks-A Combined Technique Classifying Small Molecular Structures Using a Concatenated Spectral Database, International Journal of Molecular Sciences 12 (2011) 66686684.

6. Lipinski C.A., Lombardo F., Dominy B.W., Feeney P.J., Experimental and computational approaches to estimate solubility and permeability in drug discovery and development settings, Journal of Elsevier Advanced Drug Delivery Reviews 46 (2001) 3-26.

7. Ghose A.K., Viswanadhan V.N., Wendolowski J.J., Prediction of hydrophobic (lipophilic) properties of small molecules using fragmental methods: An analysis of ALOGP and CLOGP methods, Journal of Physical Chemistry 102 (1998) 3762-3772.

8. Ghose A.K., Viswanadhan V.N., Wendolowski J.J., A knowledge-based approach in designing combinatorial or medicinal chemistry libraries for drug discovery, 1: A qualitative characterization of known drug databases, Journal of Combinatorial Chemistry 1 (1999) 5567.

9. Oprea T.I., Property distribution of drug related chemical databases, Journal of ComputerAided Molecular Design 14 (2000) 251-264.

10. Praisler M., Gosav S., Dinica R., Choosing between GC-FTIR and GC-MS spectra for an efficient intelligent identification of illicit amphetamines, Journal of Molecular Structure 887 (2008) 269-278. 